\title{
The Carotenoid pathway: What is important for excitation quenching in plant antenna complexes?
}

Kieran F. Fox ${ }^{1}$, Vytautas Balevicius ${ }^{1}$, Jevgenij Chmeliov²,3, Leonas Valkunas ${ }^{2,3}$, Alexander V. Ruban ${ }^{1}$, Christopher D. P. Duffy ${ }^{1 \#}$

${ }^{1}$ School of biological and Chemical Sciences, Queen Mary University of London, London E1 $4 N S$, UK

${ }^{2}$ Department of Theoretical Physics, Faculty of Physics, Vilnius University, Sauletekio Ave. 9, 10222 Vilnius, Lithuania

${ }^{3}$ Molecular Compound Physics Department, Center for Physical Sciences and Technology, Sauletekio Ave. 3, 10257 Vilnius, Lithuania

\#Corresponding author: c.duffy@qmul.ac.uk

\section{Abstract}

Plant light-harvesting is regulated by the Non-Photochemical Quenching (NPQ) mechanism involving the reversible formation of excitation quenching sites in the Photosystem II (PSII) antenna in response to high light. While the major antenna complex, LHCII, is known to be a site of NPQ, the precise mechanism of excitation quenching is not clearly understood. A preliminary model of the quenched crystal structure of LHCII implied that quenching arises from slow energy capture by carotenoid pigments. It predicted a thoroughly quenched system but offered little insight into the defining aspects of this quenching. In this work, we present a thorough theoretical investigation of this quenching, addressing the factors defining the quenching pathway and possible mechanism for its (de)activation. We show that quenching in LHCII crystals is the result of slow energy transfer from chlorophyll to the centrally-bound lutein carotenoids, predominantly the lut620 associated with the chlorophyll 'terminal emitter', one of the proposed in vivo pathways. We show that this quenching is rather independent of the particular species of carotenoid and excitation 'site' energy. The defining parameter is the resonant coupling between the pigment co-factors. Lastly, we show that these interactions must be severely suppressed for a light-harvesting state to be recovered. 


\section{Introduction}

The major light-harvesting pigment-protein complex of photosystem II (PSII) is LHCII, a cyclic trimer of protein sub-units that binds a densely-packed set of chlorophyll (Chl) and carotenoid co-factors. Figure 1 shows a monomeric sub-unit of LHCII with its pigment complement consisting of 8 Chls $a, 6$ Chls $b$ and four carotenoids: two centrally-bound luteins, a 9-cis neoxanthin and the peripheral, loosely-bound violaxanthin.

LHCII possesses a remarkable functional flexibility in order to regulate light-harvesting in a fluctuating light environment. In low light, it adopts an efficient light-harvesting conformation, ensuring that the PSII reaction centres (RCs) receive a consistent and sufficient supply of excitation energy. However, in high light this efficiency leads to rapid saturation of the RCs. The subsequent build-up of excitation energy in the antenna potentially leads to slowlyreversible oxidative damage to the RCs known as photoinhibition ${ }^{1}$. LHCIl, however, has the ability to respond to high light, triggered by a strong trans-membrane $\mathrm{pH}$ gradient ${ }^{2}$ generated by a high rate of photosynthetic water oxidation in the PSII RCs, switching to a photoprotective conformation. This conformation is associated with a high rate of non-radiative decay of excitation energy (quenching), meaning that excess excitation energy in the antenna is harmlessly dissipated as heat ${ }^{2}$. Despite extensive study, the nature of the quenching sites in the antenna and the precise mechanism of their formation and relaxation are ambiguous. Indeed, at time of writing there are several proposed models of the dissipative pathways in the photoprotective state, and the reader is directed to several ${ }^{3-7}$ and a recent review ${ }^{2}$.

Understanding the fine details of energy transfer and relaxation in LHCII (and LHCs generally) is currently a goal of many theoretical studies ${ }^{8-10}$. The availability of several high-resolution crystal structures ${ }^{11}$ enables an $a b$ initio approach. This is particularly relevant to photoprotective quenching since Pascal et al. ${ }^{4}$ showed that LHCIl crystals exhibit significant fluorescence quenching relative to solvated LHCII and possess many of the same spectral features as the in vivo photoprotective state. Hence, the published crystal structure represents a quenched conformation rather than the light-harvesting state. It is therefore reasonable to assume that a model of these crystal structures should capture the quenching pathway.

Structure-based models rely on quantum chemical calculations of the optical transition properties of the pigments (excitation energy and transition density) and the pairwise interaction between them. The excitation transfer and spectral dynamics are then calculated based on a specific model of the pigment-environment interaction. Novoderezhkin et al. 8,12 used a Modified Redfield approach which yielded a simultaneous fit to all steady-state and transient spectral measurements of LHCII. Later Müh et al. ${ }^{9}, 13$ incorporated a detailed description of the protein, lipid and water environment, which reproduced these spectra without the need for extensive fitting. Mennucci and co-workers ${ }^{10}$ have coupled these calculations to a Molecular Dynamics (MD) simulation, showing the importance of dynamic pigment-protein interactions in defining the function of the complex. Despite much progress, these models fail to identify a/the quenching pathway. A likely reason for this is the fact that these models treat only the $\mathrm{Chl}$ cofactors and exclude the carotenoids. Several groups have reported that energy transfer between $\mathrm{Chl}$ and the carotenoid $\mathrm{S}_{1}$ state is a defining characteristic of the quenched state ${ }^{3,5,14,15}$. Indeed this state is an attractive candidate for the quencher itself due to its unusually short lifetime, under-going non-radiative decay to the ground state in 10-20 ps ${ }^{16}$. 
Recently Chmeliov et al. ${ }^{17}$, following on from earlier work on bacterial $\mathrm{LHCs}^{18}$, produced a structure-based model of LHCII that included the carotenoids (termed an 'all pigment' model). Unlike the previous Chl-only models, this model was characterized by strong excitation quenching due to incoherent transfer of excitation energy to the $S_{1}$ state of the centrally-bound luteins. It was proposed that this pathway accounts for the strong fluorescence quenching in LHCII crystals and may reflect a/the photoprotective pathway in vivo. This model is broadly consistent with the transient absorption measurements of Ruban et al. ${ }^{5}$ which demonstrated that quenching in LHCII proceeded via incoherent Chl-to-lutein energy transfer.

Despite capturing the quenched nature of the LHCII crystal structure, the model of Chmeliov et al. ${ }^{17}$ relied on a number of arbitrary parameters and contained a number of inconsistencies. Pascal et al. ${ }^{4}$ showed that the average fluorescence decay kinetics of LHCIl crystals were mono-exponential with lifetime of $\sim 0.89 \mathrm{~ns}$, Fluorescence Lifetime Imagining (FLIM) of these crystals showed this was fairly uniform across the whole crystal with only minor variation $(0.78<\tau<1.08 \mathrm{~ns})$. This falls within the $0.2<\tau<1.5 \mathrm{~ns}$ lifetime of quenched LHCII aggregates rather than the $\tau \sim 4$ ns lifetime of unquenched solubilized trimers. Moreover, these crystals exhibited the Raman signature of neoxanthin twisting and the same fluorescence spectrum as aggregates and the PSII antenna in the photoprotective state. Later it was shown that the average lifetime had some dependence on crystal size, morphology and age $(0.4<$ $\tau<2.5 \mathrm{~ns}$ ) but the fluorescence kinetics were always mono-exponential ${ }^{19}$. The lifetime predicted by the model was $\tau \sim 80 \mathrm{ps}$, overestimating quenching when compared to even the most quenched crystals. Secondly, while Pascal et al. did not unambiguously attribute this quenching to any particular locus, species or mechanism, Ruban et al. ${ }^{5}$ showed that in aggregates the quenching site was the lutein molecule associated with the terminal emitter (lut620 in the notation of Liu et al. ${ }^{11}$ ). The Chmeliov model of the crystal predicted that both LHCII luteins (lut620 and lut621) more-or-less equally contributed to quenching. Lastly, the model predicted that the luteins functioned as irreversible traps, with the back transfer of energy from the lutein $S_{1}$ state to neighbouring Chls (de-trapping) completely absent. This is in contradiction to the work of van Amerongen et al. ${ }^{20}$ which suggests that lutein $\mathrm{S}_{1}$ to $\mathrm{Chl} a$ $Q_{y}$ energy transfer contributes significantly to the overall light harvesting efficiency of LHCII.

Concerning the quenching by both luteins, this may be an artefact of the way in which pigment geometry was treated in the model. To ensure that the non-planar distortions of the carotenoids (induced by the protein binding pocket) were maintained during geometry optimization, the dihedral angles along the backbone of the pigments were frozen at their crystal structure values. However, this process leads to artificial geometries and distortions to the pigment transition densities (see discussion) and, therefore, inter-pigment couplings.

This was partially addressed in the work of Fox et al. ${ }^{21}$ Previously, Chang et al. ${ }^{22}$ reported that there are subtle but significant differences between the conformations of lut620 and lut621 and proposed that these may manifest in different functional roles. Fox et al. ${ }^{21}$ studied the effect of these differences on Chl-lutein $S_{1}$ coupling by adopting the more accurate optimization scheme of Götze, Kröner and co-workers ${ }^{23,} 24$ Within this scheme pigment geometries are optimized within a static 'cage' of molecular fragments representing the local environment of each pigment (see methods section). This study showed that such small structural differences could have a significant effect on resonant couplings, with the lut620chla612 coupling stronger than lut621-chla60321. It was therefore hypothesized that lut620 may be more accessible to $\mathrm{Chl}$ excitations than lut621, accounting for the observation of 
Ruban et al. ${ }^{5}$ that lut620 is the exclusive quencher. The effect could influence the $\mathrm{S}_{1}$ energies which would in turn influence energy transfer rates.

The irreversibility of the lutein quenching traps in Chmeliov et al. ${ }^{17}$ was due primarily to how the Chls-to-carotenoid transfer rates were computed (see methods). According to classical Förster theory, this transfer rate is proportional to the spectral overlap of donor fluorescence and acceptor absorption. Since the carotenoid $S_{1}$ state is optically forbidden, Chmeliov et al. replaced this absorption spectrum with a density of states (DOS) distribution ${ }^{25}$. This DOS distribution was obtained by fitting a single Gaussian lineshape function to the two-photon absorption spectrum of lutein in octanol ${ }^{26}$, characterized by an over-damped Brownian oscillator spectral density. Fitting the calculated spectral line to this very broad two-photon spectrum required an enormous reorganisation energy, $\lambda_{0}$, of $3450 \mathrm{~cm}^{-1}$. This has the effect of assigning an unphysically large Stokes shift, $2 \lambda_{0}$, to the carotenoid $S_{1}$ state meaning that the carotenoid-to-Chl energy transfer is entirely thermodynamically suppressed. We here contend that it is highly unlikely that this two-photon spectrum originates from a single homogeneously-broadened transition. Indeed, previous empirical models of energy relaxation in carotenoid dyads have assumed a reorganization energy as low as $300 \mathrm{~cm}^{-1} 27-29$. Moreover, it has been shown in studies on bacterial LHCs that such a simplified approach to calculating donor and acceptor overlap is inaccurate. We here propose a more realistic 'lineshape' for the the $\mathrm{S}_{1}$ transition based on the two-photon absorption measurements of Walla et al. ${ }^{26}$.

Another relatively difficult parameter to obtain, due to the dark nature of the $S_{1}$ state, is its excitation energy. A review by Polivka and Sundstrom ${ }^{30}$ reported huge ranges $\left(\Delta \mathrm{E}_{\mathrm{S} 1} \sim 900\right.$ $\mathrm{cm}^{-1}$ ) in carotenoid $S_{1}$ energy depending on the method of measurement (two-photon, excited state absorption, fluorescence, etc.) and experimental conditions. In the Chmeliov et al. study ${ }^{17}$, the $S_{1}$ transition energies of the four LHCII carotenoids were optimized to maximise quenching and establish a lower limit on LHCII lifetime. However, due to assumption of a single, ultra-broad DOS distribution assigned to the carotenoids, the calculated Chl-to-lutein energy transfer rates were largely insensitive to these energies.

Although the model of Chmeliov et al. gave a preliminary molecular picture of quenching in LHCII, it did not address which parameters were important to this mechanism. How sensitive is such a quenching mechanism to the chosen parameters? What are the defining properties of an effective quencher? How may such a quenching mechanism be deactivated in the lightharvesting state? In this work, we present a structure-based model of energy transfer and quenching within a monomeric sub-unit of LHCII. As in Chmeliov et al. ${ }^{17}$, we pay particular attention to the poorly-understood roles of Chl-carotenoid interactions. While the Chmeliov et al. paper gave a first investigation of these dynamics, here we present a more thorough consideration of how Chl-carotenoid coupling, carotenoid excitation energies and pigment geometries define the quenching within the system. It must be possible to reduce the involvement of the carotenoids (which in our model bring about the quenched state) to such a degree as to allow light harvesting: an increase of the mean excitation lifetime to the in vivo values to $2 \mathrm{~ns}$. By altering the energies and couplings in realistic ways, we can determine which of them are likely to affect the transfer of energy to the carotenoid in the real system. 


\section{Methodology}

\subsection{Geometry optimisations}

The crystal structure of LHCII (PDB ID: 1RWT) gives a good representation of the bulk structure, but due to limited resolution, there are small errors in important quantum chemical parameters, such as bond length and angle. The pigments therefore need careful optimisation before carrying out subsequent calculations ${ }^{31}$. within an explicit cage of the surrounding pigment-protein environment (see Supp. Figs. 1-4) following the method of Gotze and Kroner $^{23,24}$. During such a procedure the central pigment, optimized using density functional theory (DFT) with the B3LYP ${ }^{32}$ exchange correlation functional and the $6-31 G^{*}$ basis set ${ }^{33}$, is not subject to any intrinsic geometrical restrictions. The environmental cage typically contains 500-1200 atoms and therefore, to spare computational expense, is modelled with the semiempirical PM6 Hamiltonian ${ }^{34}$. To maintain the geometry of the cage during optimization of the central pigment, the coordinates of all heavy atoms in the cage are frozen.

\subsection{Excited states and inter-pigment coupling}

As in previous studies, we employ a full configuration interaction $(\mathrm{Cl})$ calculation within a complete active space (CAS) of orbitals obtained using the semi-empirical AM1 Hamiltonian ${ }^{35}$ (AM1-CAS-CI), as implemented by the MOPAC2012 package ${ }^{36}$. Kusumoto et al. ${ }^{37}$ showed that this method predicts an $S_{1}$ state with the correct optical properties and two-electron character ${ }^{38}$ and is consistent with more expensive $a b$ initio methods, such as SAC-CI and CASSCF. As in Chmeliov et al..$^{39}$, we used a CAS of 6 orbitals (HOMO-2 to LUMO+2) and for the sake of consistency we used the same method to calculate the Chl $\mathrm{Q}_{\mathrm{y}}$ transitions.

Inter-pigment excitation energy transfer is mediated by resonance interactions between the electronic transitions of a donor molecule $(D)$ and an acceptor molecule $(A)$, as characterized by the inter-molecular transfer integral,

$$
W_{D A}=J_{D A}-K_{D A}
$$

where $J_{D A}$ is the Coulomb interaction and $K_{D A}$ is the exchange interaction. The exchange part is determined by atomic orbital overlap and as such falls off exponentially with increasing intermolecular distance. It is therefore commonly neglected ${ }^{9,13}\left(W_{D A} \approx J_{D A}\right)$, an assumption that we also make in this work (although the validity of this for interactions involving a dark state are discussed later and in the supplementary material). Formally, the Coulomb transfer integral is an interaction between two spatially extended molecular transition charge densities. Various schemes exist to evaluate this interaction approximately, such as the point-dipole and transition monopole/charge approximations, although the latter tend to overly simplify the real transition density while the former is entirely inappropriate for the closely-packed interior of LHCII. A very accurate method is the transition density cube (TDC) approach, in which the true molecular transition densities are approximated as a fine 3-dimensional grid of charge elements (cubes),

$$
M_{D / A}\left(x_{1}, y_{1}, z_{1}\right) \approx \int_{x_{1}}^{x_{1}+\delta x} \int_{y_{1}}^{y_{1}+\delta y} \int_{z_{1}}^{z_{1}+\delta z} d x d y d z \Psi_{G S} \Psi_{E X}^{*}
$$


(2)

where $\Psi_{G S}$ and $\Psi_{E X}$ are the ground and excited state wave functions respectively. $J_{D A}$ is therefore approximated as the sum of pairwise Coulomb interactions between the cubes,

$$
J_{D A} \approx J_{D A}^{T D C}=\frac{e^{2}}{4 \pi \varepsilon \varepsilon_{0}} \sum_{\substack{i \in D \\ j \in A}} \frac{M_{D}(i) M_{A}(j)}{\left|\vec{r}_{l}-\vec{r}_{j}\right|}
$$

(3)

As the size of the volume elements decreases, $J_{D A}^{T D C}$ approaches the true $J_{D A}$. As in previous studies, we choose a grid size of $0.25 \AA$, which gives an average relative error of $<0.1 \%$. The TDCs were calculated using the code of Bricker et al. ${ }^{40}$ and the couplings using software developed by Krueger et al. ${ }^{41}$

Transition dipole moments as calculated by quantum chemistry are typically over-estimated with respect to the experimental values so the TDCs must be rescaled accordingly. The TDCs of the $14 \mathrm{LHCll}$ Chls were rescaled so that their average $\mathrm{Q}_{\mathrm{y}}$ transition dipole moment matched the vacuum-extrapolated values reported by Knox and Spring ${ }^{42}$. Since the carotenoid $S_{1}$ state is dipole forbidden, no such scaling was applied for Cars (the validity of this is discussed later). To account for the solvent screening of the protein environment, a relative dielectric constant of $\varepsilon=2$ was assumed for pigment interactions, unless the inter-pigment association was so close as to exclude the solvent (as in previous models).

\subsection{Energy transfer and relaxation}

The formalism adopted for modelling the energy transfer dynamics of the LHCII monomer is essentially the same as in Chmeliov et al. ${ }^{17}$. The full excitonic Hamiltonian of the LHCII monomer is defined,

$$
\widehat{H}=\sum_{m=1}^{N} E_{m}|m\rangle\left\langle m\left|+\sum_{m \neq n}^{N} J_{m n}\right| m\right\rangle\langle n|
$$

(4)

where $N=18$ is the number of pigments in the monomer ( 8 Chls $a, 6$ Chls $b$, and 4 carotenoids), $E_{m}$ is the energy of the $Q_{y} / S_{1}$ state (site energy) of the $m^{\text {th }}$ pigment, and $J_{m n}$ is the coupling between the $m^{\text {th }}$ and $n^{\text {th }}$ molecules. For the Chls, the site energies are taken from the Müh et al. ${ }^{13}$ (as in previous models). For the carotenoids, the site energies are not well defined. Unlike in Chmeliov et al. ${ }^{17}$, we do not simply pick some optimum values, but rather treat them as a (relatively) free parameter. We assume that the $S_{1}$ energies obtained from the AM1-CAS-CI calculation are correct only in a relative sense, but the absolute energies are undefined with respect to a single scaling factor $\alpha$, which is treated as a (relatively) free parameter:

$$
E_{m}=\alpha E_{m}^{c a l c}
$$

In the review of Polivka and Sundstrom ${ }^{30}$ several measurements of the lutein $\mathrm{S}_{1}$ energy are reported, ranging from $14050 \mathrm{~cm}^{-1}$ (excited state absorption ${ }^{43}$ ) to $<15300 \mathrm{~cm}^{-1}$ (two photon 
absorption ${ }^{26}$ ). We have decided to use the value of $15100 \mathrm{~cm}^{-1}$ for lutein in native LHCII, obtained using two photon absorption, as this the vertical excitation most appropriate in describing the acceptor behaviour of the carotenoids. However, in order to reflect the uncertainty, we vary the energies using the global scaling factor: $\alpha$.

In the Förster regime the rate constants for energy transfer from the $n$th to the $m$ th pigment are defined as

$$
k_{m n}=2\left|J_{m n}\right|^{2} \operatorname{Re} \int_{0}^{\infty} A_{m}(t) F_{n}^{*}(t) \mathrm{d} t
$$

(6)

where

$$
A_{n}(t)=\mathrm{e}^{-\frac{\mathrm{i} E_{n} t}{\hbar}-g_{n}(t)}
$$

and

$$
F_{m}(t)=\mathrm{e}^{-\mathrm{i}\left(\frac{E_{m}-2 \lambda_{m}}{\hbar}\right) t-g_{m}^{*}(t)}
$$

(8)

are the acceptor absorption (7) and donor fluorescence (8) response functions in the time domain, and $2 \lambda_{m}$ is the Stokes shift. The line-broadening function is defined as

$$
g_{n}(t)=\int_{0}^{\infty} \frac{\mathrm{d} \omega}{\pi \omega^{2}} C_{n}{ }^{\prime \prime}(\omega)\left[(1-\cos (\omega t)) \operatorname{coth}\left(\frac{\hbar \omega}{2 k_{B} T}\right)+\mathrm{i}(\sin (\omega t)-\omega t)\right]
$$

(9)

For the Chls functions $A_{n}(t)$ and $F_{m}(t)$ correspond to physical spectra, defined by the analytical spectral density developed by Renger et al. ${ }^{44}$,

$$
C_{n}{ }^{\prime \prime}(\omega)=\frac{\pi S_{0} \omega^{5}}{s_{1}+s_{2}} \sum_{i=1}^{2} \frac{s_{i}}{7 ! 2 \omega_{i}{ }^{4}} e^{-\sqrt{\frac{\omega}{\omega_{i}}}}
$$

where $S_{0}=0.5, s_{1}=0.8, s_{2}=0.5, \omega_{1}=0.56 \mathrm{~cm}^{-1}$ and $\omega_{2}=1.94 \mathrm{~cm}^{-19,13,45}$.

The carotenoids spectral density is newly generated from the two-photon absorbtion spectra of Walla et al. ${ }^{26}$ and is described in section 2.4 below.

With the rate constants now defined, the evolution of the system is described by the Pauli Master Equations,

$$
\frac{\mathrm{d}}{\mathrm{d} t} P_{n}(t)=\sum_{m \neq n}\left[k_{n m} P_{m}(t)-k_{m n} P_{n}(t)\right]-\left(k_{\mathrm{F}}+k_{\mathrm{NR}}\right) P_{n}(t)
$$


where $P_{n}(t)$ is the time-dependent probability for the excitation to reside on the $n$th pigment, $k_{\mathrm{F}}$ is the fluorescence rate and $k_{\mathrm{NR}}$ is the rate of non-radiative decay. For the Chls we assume $k_{\mathrm{F}}^{-1}=16 \mathrm{~ns}$ and $k_{\mathrm{NR}}^{-1}=5.3 \mathrm{~ns}$ (consistent with the $4 \mathrm{~ns}$ lifetime of $\mathrm{Chl}$ in solution ${ }^{46}$ ). For the carotenoids, the parameters $k_{\mathrm{F}}^{-1}=\infty$ and $k_{\mathrm{NR}}^{-1}=10 \mathrm{ps}$ were assumed as in the previous study ${ }^{17}$ and is supported experimentally ${ }^{43}$. The former assumption is based on the dark nature of the $S_{1}$ state and the latter comes from measurements of the $S_{1}$ lifetime in recombinant LHCII.

Lastly, excitonic delocalization was also accounted for. Whenever the calculated hopping times (inverse rates) were less than $1 \mathrm{ps}$, it was assumed that these pigments form an 'excitonic cluster'. The excitation dynamics in the LHCIl monomer was assumed to be hierarchical, with instantaneous equilibration within these excitonic clusters and incoherent, Förster-type transfer between clusters and isolated pigments. It has been shown that this cluster (or domain) approach reproduces the dynamics of more detailed approaches such as combined Modified Redfield-Generalized Förster theory ${ }^{17,47}$.

\subsection{Carotenoids spectral density}

In order to obtain the spectral density of the Cars, we reconsidered the two-photon absorption spectrum of lutein by Walla et al. ${ }^{26}$. It has been shown that various spectroscopic signals of carotenoids (e.g., absorption, induced absorption from $\mathrm{S}_{1}$ ) can be very accurately described by considering vibronic transitions that include two high-frequency carbon-carbon stretching modes $^{48}$. We therefore start by constructing an ansatz-spectral density that includes two underdamped terms to describe the two high-frequency vibrations ${ }^{49}$ plus an overdamped term describing the remaining degrees of freedom:

$$
C(\omega)=\sum_{i=1,2} 2 \lambda_{i} \frac{\omega \omega_{i}^{2} \gamma_{i}}{\left(\omega^{2}-\omega_{i}^{2}\right)^{2}+\omega^{2} \gamma_{i}^{2}}+2 \lambda_{0} \frac{\omega \gamma_{0}}{\omega^{2}+\gamma_{0}^{2}}
$$

Next, we calculate the absorption line-shape $A_{n}(t)$ as given in Eq. (7). Then we calculate the absorption spectrum via Fourier transform of $A_{n}(t)$ and fit the two-photon absorption data so that the slopes would yield best visual fit of the outlying points. The resultant spectrum is shown in Fig. 2 along with the single-photon absorption obtained from the same ansatz (with different parameters) for comparison. We used the values of the single- and double-bond stretching frequencies, $\omega_{2}$ and $\omega_{1}$ accordingly, determined by resonance Raman spectroscopy ${ }^{50}$. For lutein the reported values are $\omega_{1}=1522 \mathrm{~cm}^{-1}$ and $\omega_{2}=1156 \mathrm{~cm}^{-1}$, for neoxanthin $\omega_{1}=1530 \mathrm{~cm}^{-1}$ and $\omega_{2}=1156 \mathrm{~cm}^{-1}$, for violaxanthin $\omega_{1}=1524 \mathrm{~cm}^{-1}$ and $\omega_{2}=1156 \mathrm{~cm}^{-1}$. The following parameters are obtained from the two-photon absorption fitting: $\lambda_{1}=\lambda_{2}=900 \mathrm{~cm}^{-1}, \gamma_{1}=\gamma_{2}=300 \mathrm{fs}$ for the underdamped part; $\lambda_{0}=450 \mathrm{~cm}^{-1}, \gamma_{0}=$ $53 \mathrm{fs}$ the overdamped part.

\section{Results}

\subsection{Geometry optimisations and excited states}

The resulting geometries were all checked against the original conformations from the crystal structure ${ }^{11}$. They were found to be in close agreement but with some clear alterations. The 
optimisations all tend towards the planar (vacuum) structure but still retain some distortions and individuality. Importantly the optimized structures are consistent with the original crystal structure within the resolution of the latter. The optimized geometries of the carotenoids (alongside starting geometries) are listed in Supp. Figs. 1-4.

A comparison of the calculated and measured energies, and dipole moments for the $S_{1}$ and $Q_{y}$ states of the carotenoids and chlorophylls respectively are presented in Table 1. AM1$\mathrm{MRCl}$ yields a correct description of the carotenoid $S_{1}$ state which is predicted to be essentially optically forbidden and possess a strong two-electron character (predominantly double HOMO to LUMO) ${ }^{51}$. The excitation energies are somewhat over-estimated compared to the ranges reported experimentally (values reported in table 1). In Table 1 we also compare our calculated energies to those obtained by Andreussi et al. ${ }^{52}$ via a Multi Reference Configuration Interaction calculation on a set of orbitals obtained using DFT (DFT/MRCI). These energies are also overestimated but to a lesser degree. More importantly, the energies calculated here differ from Andreussi et al. by a single scaling factor of $\sim 1.1$ (1.14 for lutein and violaxanthin, and 1.11 for neoxanthin). Neglecting solvent effects, we contend that our calculated energies are reasonable in a relative sense and the use of a single scaling factor in the rate calculations is justified. For the Chls, Table 1 reports average values for the same quantities and we see that there is reasonable agreement with experiment for excitation energies and dipole moments (apart from the over-estimate of the absolute energy, inherent to calculations of this nature).

The 'cage' optimisation method was preferable to previously used methods such as dihedral freezing or modulating the bond parameters during post-optimisation as there are no artificial distortions to bond lengths or calculated transition densities/dipoles. The most important of these distortions is the bond length alternation (BLA) pattern along the conjugated backbone of the carotenoid. Studies by Mennucci and co-workers ${ }^{10,53}$ have shown that the $S_{1}$ dipole is highly sensitive to this. We found that dihedral freezing produced a significant deviation from the bond alternation pattern of vacuum optimised carotenoids (Supp. Fig. 5). The vacuum optimised Lut620 geometry gave $B L A=0.0030 A$ which is the same (to 2 s.f.) to our 'cage optimised' structure $B L A=0.0030 \AA$, whereas the dihedral freezing gave $B L A=0.0032 A$. This has the expected consequences on the dipole lengths of the two distorted structures. The cage optimised dipole is low at $\mu=0.17 D$ whereas the dihedral frozen structure gives an elevated dipole length of $\mu=0.49 D$.

\subsection{Couplings and transfer rates}

The exciton Hamiltonian (in the site basis) is presented in Table 2, where the new coupling values, $J_{m n}$, based on the cage-optimized pigments (lower left triangle), are compared with the values previously used by Chmeliov et al. ${ }^{17}$ (upper right triangle). The site energies, $E_{m}$, are the diagonal elements with $\mathrm{Chl}$ energies taken from Müh et al. ${ }^{13}$ The carotenoid site energies are the unscaled calculated values. Compared to Chmeliov et al., there is an overall reduction in the coupling strengths. This is attributable to the more relaxed, slightly more planar geometries obtained from the lack of artificial geometric restraints. As a result, the new couplings match those calculated for planar Chls (using the transition atomic charge method) more closely. Broadly, the Chl-Chl couplings remain the same as in previous studies ${ }^{9,13,17}$. We see several strongly-coupled Chl pairs: chla611-chla612 (part of the terminal emitter domain with chla610); chla604-chlb606; and chla603-chlb609. 
The Chl-carotenoid couplings also broadly match those of Chmeliov et al. ${ }^{17}$, being vanishingly small for all but a few very closely associated pigment pairs. Even in the case of these pairs, the resulting coupling is around an order of magnitude smaller than the Chl-Chl couplings (see discussion). Lut620 is only significantly coupled $\left(6 \mathrm{~cm}^{-1}\right)$ to Chla612 and this is mirrored $\left(7 \mathrm{~cm}^{-}\right.$ ${ }^{1}$ ) in the broadly structurally similar lut621-chla603 (see Fig. 1) domain. The peripherally-bound vio622 is weakly-coupled to chla614 $\left(5 \mathrm{~cm}^{-1}\right)$ while neo623 is coupled to chla604, chlb606 and chlb608 (6, 7 and $9 \mathrm{~cm}^{-1}$, respectively). Significantly, we see that the Lut-Chl couplings are $40-50 \%$ weaker than the values given in Chmeliov et al. ${ }^{17}$ We may tentatively attribute this to the optimization scheme and the absence of unphysical bond and transition density distortions ${ }^{21}$. Unexpectedly, the neo623-Chl couplings have increased by a similar amount. The reason for this is not immediately obvious. However, there are subtle differences in geometry between our optimized structures and the frozen structure of Chmeliov et al. (both for the Chl's and the carotenoids). While this has little effect on the strongly optically allowed Chls, Fox et al. ${ }^{21}$ showed that Chl-carotenoid couplings are highly sensitive to alterations in inter-pigment associations and intra-pigment conformations (see discussion).

The dynamics of the Chl pool are essentially identical to those reported in Chmeliov et al., given similar couplings and spectral overlaps. As before, we identified two excitonic domains: chlb603-chla604 and the terminal emitter chla610-chla611-chla612 domains. The interpigment/domain hopping times do not differ from those reported in Chmeliov et al. ${ }^{17}$ in any meaningful way.

The Chl-carotenoid dynamics naturally depends on the site energy scaling factor, $\alpha$. Fig. 3 shows the chlorophyll-carotenoid interaction we believe to most important due to the proposed quenching pathway, 17,39 as well as particularly fast transfer times. Hopping time, $k_{m n}^{-1}$, is plotted as a function of $E_{L u t 620}$ for each interaction in both directions with the carotenoidchlorophyll transfer being presented in a faded version of the same colour as the reverse. In the review of Polivka and Sundstrom ${ }^{30}$, two energies of lutein, using the chosen method of two-photon absorbtion, are reported $15100 \mathrm{~cm}^{-126}$ and $15300 \mathrm{~cm}^{-154}$. This method was chosen over fluorescence, Raman and excited state absorption as it probes the vertical transition. The lower value of $15100 \mathrm{~cm}^{-1}$ was chosen as the more recent measurement but a range of values around this was taken to account for the uncertainty. We chose a range of $+/-500 \mathrm{~cm}^{-1}$ to properly encompass the range of $S_{1}$ energies reported for lutein ${ }^{30}$. We then use our calculated ratio of $S_{1}$ energies to scale the other carotenoids to this measurement. As stated above we are confident in this ratio of energies as it compares favourably to those calculated using a much more computationally expensive method by Andreussi et al. ${ }^{52}$.

The fastest transfer displayed in Fig. 3 is between neoxanthin and Chlb608, however, this interaction is not important for quenching as there is very little excitation density on this chlorophyll. This is because of the higher $Q_{y}$ energy of the chlorophyll bs compared to chlorophyll a, as well as the rapid thermal equilibration. This interaction is likely to be a light harvesting one as the short hopping times can compete with the $S_{1}$ dissipation lifetime. The fastest transfer to neoxanthin is from Chla604 and is shown to be much slower than transfer to the two luteins. Transfer to violaxanthin was omitted from this graph as it is so much slower than these interactions, it was deemed to be unimportant for NPQ as well as light harvesting. The hopping times between Lut620 and Chla612 is very similar to between Lut621 and Chla603 which is to be expected due to the similarity between the two sites. 
Chmeliov et al. ${ }^{17}$ predicted deep traps (carotenoids) that had virtually no transfer back to the chlorophylls from the carotenoids. The irreversibility of the quenching reported by them is likely an unphysical artefact of the massive carotenoid reorganisation energy in the model of 3540 $\mathrm{cm}^{-1}$ leading to an unrealistically large stokes shift. Our new spectral density shows significantly larger carotenoid to chlorophyll transfer. This is in line with the work of van Amerongen et al. ${ }^{20}$ who showed that carotenoid $\mathrm{S}_{1}$ to $\mathrm{Chl} \mathrm{Q}_{\mathrm{y}}$ transfer contributes significantly to light-harvesting. However, we note that even in the case of reversible carotenoid traps the system is highly quenched as it is difficult to outcompete the 10ps rate of decay from the $S_{1}$ state.

The minimum hopping times naturally occur at the point of resonance between chlorophyll and carotenoid. At this point the Förster picture of energy transfer is not valid, and excitonic delocalization predominates. However, the excitonic picture is only strictly valid if

$$
\Delta E_{m n}=\left|E_{m}-E_{n}\right| \ll 2 J_{m n}
$$

Due to the small resonance coupling this window is very small $\left(\Delta E_{m n}<12 \mathrm{~cm}^{-1}\right)$ and dephasing is likely to be very fast. Regardless of $E_{\text {Lut620 }}$, we see that the hopping time is slow $\left(k^{-1}>30 \mathrm{ps}\right)$.

\subsection{Lifetime and quenching pathways}

If the carotenoids are neglected in our model, the mean excitation lifetime of the LHCII monomer is $\tau=4 \mathrm{~ns}$, due to the intrinsic $4 \mathrm{~ns}$ lifetime of isolated $\mathrm{Chl}^{46}$. This lifetime is consistent with that of the detergent solubilized LHCII trimer and monomers which serve as a model for the unquenched (light-harvesting) state. As in the previous work by Chmeliov et al. ${ }^{17}$, inclusion of the carotenoids yields a system that is quenched.

Fig. 4a shows the dependence of the mean excitation lifetime, $\tau$, on the carotenoid $\mathrm{S}_{1}$ energy (represented by Lut620). Fig.2 also shows the lifetime using the simple over-damped spectral density from the previous paper ${ }^{17}$ with their reorganisation energy as well as the lowest reported for carotenoids ${ }^{29}$. This is simply to illustrate the changes due to our new spectral density. The longer lifetime is likely a result of the increased carotenoid to chlorophyll transfer competing with $S_{1}$ thermal dissipation.

We can see that there is little variation in lifetime across this range and there is certainly no sharp transition from a dissipative to a light harvesting state. From this we can say that slow Forster type transfer to the carotenoids is enough for quenching in LHCll. This is comparable to the quenched lifetime component of $\tau=130 \mathrm{ps}$ measured for quenched LHCIl aggregates by Ruban et al. ${ }^{5}$. Our calculated lifetime at an $S_{1}$ energy of $15100 \mathrm{~cm}^{-1}$ is $\tau=170 \mathrm{ps}$. It must be noted, however, that at all energies our lifetime is consistently shorter than the $0.78<\tau<$ 1.08 ns crystal lifetime measured by Pascal et al. ${ }^{4}$ (see the discussion section).

Fig. $4 \mathrm{~b}$ indicates how this excitation quenching is partitioned between the four carotenoids as a function of carotenoid site energy scaling (represented by lut620 site energy). We can see that all carotenoids contribute to quenching which is expected as we have reported transfer to the carotenoid in all cases. However, the contribution from violaxanthin is very small, partially due to the lack of proximal chlorophylls leading to smaller vio-chl couplings as well as the 
larger $S_{1}$ energy compared to lutein making the transition energetically unfavourable. This is in accordance with the experiments of Ruban et al. ${ }^{5}$. We also see that the luteins contribute the most to quenching as they are situated in the centre of the protein, surrounded by chlorophylls and they have a lower $S_{1}$ energy, closer to that of the chlorophyll $Q_{y}$ band. However, in both cases we note that the coupling is the primary factor for transfer as there is little variation across the range of energies. Lut621 contributes less to quenching than Lut620 at all energies. Because there is little difference in both $S_{1}$ energy $\left(E_{L u t 620} / E_{\text {Lut621 }}=0.99\right)$ and coupling (table 1), the mentioned difference in quenching efficiency mostly arises from the fact that lut620 is associated with the Chl energy sink in LHCII (the terminal emitter domain). Finally, we note that neoxanthin contributes a significant (though smaller than the luteins) amount to quenching due to the coupling between $\mathrm{Neo}$ and Chla604. As mentioned above, the very large couplings between Neo and Chlb606 and Chlb608 are not significant for NPQ. These results predict a quenching pathway broadly similar to that suggested by Ruban et al. ${ }^{5}$, although our calculations predict that lut620 is the predominant rather than the sole quencher. It appears that all that is needed for quenching is a carotenoid to be in close proximity to a low energy chlorophyll.

\subsection{Variations in Chl-carotenoid coupling}

Our model indicates that, for the generous range of realistic carotenoid site energies, these small Chl-carotenoid couplings result in quenching. Moreover, the overall lifetime of the system is not particularly sensitive to the carotenoid site energy (in all the cases, the lifetime lies within the interval $\tau=163-187 \mathrm{ps}$ ). The last aspect we examine is how the strength of the Chl-carotenoid couplings determines the extent of quenching. Fig. 5 shows various ad hoc modified coupling schemes as a function of the carotenoid $S_{1}$ energy scaling factor. Fig. $5 a$, scheme A represents the original scheme (Table 2). Scheme B corresponds to all $J_{\text {Chl-Car }}$ couplings scaled by a factor 0.2 . This results in a 3- to 6-fold increase in the overall excitation lifetime of the system, resulting in lifetimes close to those reported for light harvesting LHCII within the membrane. Fig. $5 \mathrm{~b}$ shows that such a drastic reduction in coupling is necessary due to the steep relationship between the couplings and the system lifetime.

Fig. 5a, scheme C, D and E correspond to schemes in which either lut620, lut621 or neo623 has been completely decoupled. We do not consider the decoupling of vio622 as this carotenoid contributes very little to the overall quenching. E corresponds to the decoupling of neo623, which has very little effect on the overall quenching in the system. This is expected, given that it contributes little to the overall quenching in this energetic region. Interestingly, the decoupling of one of the two luteins (C, lut620 and D, lut621) also has little effect, implying both pathways contribute to quenching and can serve as the main pathway in the event of decoupling of the other. Indeed, the decoupling of both luteins $(\mathbf{F})$ still results in a surprising level of quenching, primarily from the neoxanthin. This shows that if a bulk protein reorganisation, causing a change in the carotenoid-chlorophyll dynamics, is responsible for quenching, it needs to have an affect on both luteins as well as neoxanthin. Indeed Fig. $5 \mathrm{~b}$ shows that all interactions must be completely abolished if we are to recover the 4 ns lifetime of isolated, solubilised LHCII trimers.

\section{Discussion}

The purpose of this model was to attempt to produce a physically realistic theoretical picture of quenching in LHCll crystals. Moreover, we wanted to investigate which parameters are 
important for defining this quenching. As with the previous model ${ }^{17}$ we have shown that inclusion of the chlorophyll $Q_{y}$-carotenoid $S_{1}$ interaction leads to profound excitation quenching. Firstly, we have replaced the overly simplistic $S_{1}$ 'lineshape' of the previous model with a more physically realistic description of the vibronic structure of the transition. While this did not have a significant effect on the overall level of quenching it removed the problematic irreversible trapping that characterized the previous model. Indeed, we see that the rates of forward and backward transfer are broadly comparable. The fact that energy transfer from the $S_{1}$ state to the chlorophyll pool is possible is a new feature of this model and in line with observations of this light-harvesting pathway in LHCII.

As in the previous model the over-all level of quenching appears to be insensitive to the $S_{1}$ site energy, arising from the broad nature of the $S_{1}$ transition. We have to acknowledge that we have fixed the relative energies of the carotenoids according to the vacuum energies calculated by ourselves and Andreussi et al..$^{52}$ and there will likely be some tuning of these relative energies due to the protein. However, we do not see any large changes to the relative contributions of each carotenoid to the overall quenching in the range of energies considered. Moreover, we see that the individual transfer rates are also rather insensitive to the changes in energy. If we consider our $S_{1}$ lineshape function and the assumption of Förster transfer to be reasonable then site energy is not critical to the quenching mechanism within the crystal. Indeed, we see no sharp, 'gearshift' type transition between a quenched and an unquenched state.

A crucial parameter appears to be the chlorophyll-carotenoid couplings, which appear to be defined largely by the separation and relative orientation of the chlorophyll-carotenoid pair. Even for very-closely associated pairs we see that the couplings are very weak. The reason for this is the rather specific way in which the $S_{1}$ transition is 'dark'. As discussed by Ritz et al. ${ }^{18}$ the $S_{1}$ state is optically forbidden due to two symmetries: inversion and particlehole/alternancy. The former only (approximately) applies for all-trans isomers and although its renders the $S_{1}$ state dipole forbidden it does not preclude strong higher-order Coulomb interactions at moderately close inter-molecular distances. The latter is only an exact symmetry of some m-electron models of ideal polyene but alternacy labels are convenient short-hand for the importance of dynamic electron correlations to a particular state. '-' states such as $S_{1}\left(2 A_{g}{ }^{-}\right)$have a strong two-electron character while ' + ' state such as $S_{2}$ or the inversion forbidden cis-band states $\left(\mathrm{A}_{\mathrm{g}}{ }^{+}\right)$are one-electron excitations. This property arises from the bond-alternation of the conjugated chain rather than overall spatial symmetry and explains why the $S_{1}$ state of 9-cis neoxanthin is still resolutely forbidden. The two-electron character profoundly limits the way in which the $S_{1}$ state can interact with other chromophore transitions. Essentially, pure two-electron excitations are associated with zero transition density and the predominantly two-electron $\mathrm{S}_{1}$ state has a vanishingly small transition density. It is this that ensures very weak couplings even at very close inter-molecular distances. It is possible that both the neglected exchange and overlap interactions in our model may contribute to the overall coupling. However, both of these interactions are similarly limited by the two-electron character of the $S_{1}$ transition, with the overlap interaction likely extremely so (for the reader not familiar with these arguments a simple mathematical discussion is included in the supplementary material). Moreover, it has been shown that in bacterial systems only the Coulomb interaction makes a significant contribution to energy transfer to or from the carotenoid $S_{1}$ state. We therefore hypothesize that both exchange and overlap will be small 
corrections to our calculated couplings. However, this is still a worthy avenue of further research.

The slow nature of the quenching places an additional restriction on the contribution of a particular carotenoid. Despite the fact that neoxanthin is as coupled to the chlorophyll pool as the central luteins its predominant association with chlorophyll $b$ means it is partially outcompeted by fast equilibration of energy across the chlorophyll a pool.

Our model presents a picture of quenching of quenching in LHCIl crystals in which the carotenoids, particularly the centrally-bound luteins, slowly trap but rapidly quench excitation energy. Recently, Belgio et al. reported the economic nature of photoprotection. Analysis of the fast fluorescence induction of PSII in high and low light showed that the quenching traps present in the antenna do not compete with excitation trapping by the RCs. They proposed that this was the result of a slow, carotenoid-mediated quenching pathway within the LHCII antenna. More recently, Chmeliov et al. analysed the temperature dependence of the fluorescence kinetics of quenched LHCIl aggregates. They showed that these kinetic can be explained by the presence of a sub-population of quenched LHCll monomer, characterized by slow (50-100 ps) excitation trapping by a dark molecular state. The carotenoid-mediated quenching in our model seems to fit these observations of slow, dark quenchers. With regard to the quenching seen in LHCII crystals, Pascal et al. did not attribute this to any particular quencher but showed that crystallization is associates with some movements in both the lutein and neoxanthin domain, the domains responsible for quenching in our model.

There are however, some limitations to our model that must be discussed. Firstly, our calculated lifetime ( $\tau>170 \mathrm{ps)}$ is consistent with strongly-quenched LHCII aggregates rather than the crystals measured by Pascal et al. ${ }^{4}(\langle\tau\rangle=890 \mathrm{ps})$. There could be several reasons for this. The most obvious source of error is that we have a systematic over-estimate of the chlorophyll-chlorophyll coupling. Fig. $4 \mathrm{~b}$ shows that a factor of 2 would be sufficient to account for this deviation. Some over-estimate is very likely since we are unable to apply the post factum scaling of the transition density to the forbidden $S_{1}$ state. The neglect of the exchange and overlap contributions is most likely far less important than this error ${ }^{18}$. If this is the case then we must acknowledge that the crystal represents at partially-quenched system, which in itself is reasonable since it was crystalized with violaxanthin instead of zeaxanthin which is known to repress (but not exclude) quenching even in aggregates ${ }^{55}$. We must note however, that even in this case our model has shown that any kind of coupling to the $S_{1}$ state of lutein or neoxanthin is associated with a significant decline in the lifetime of the complex.

A second possibility is that there is some heterogeneity in the crystals with a mixture of quenched and unquenched sites present throughout. So long as they all remain mutually connected the quenched sites will be able to trap energy from its unquenched neighbours and the overall fluorescence kinetics will remain mono-exponential. This is collective behaviour observed recent in aggregates by Chmeliov et al. ${ }^{17}$, in which quenching is attributed to a 15$20 \%$ sub-population of quenched (50-100 ps) monomers. This would require very for the difference between the quenched and unquenched sites to fall within the resolution of the crystal and indeed van Oort et al. reported that volume changes of as little as $0.006 \%$ are efficient to induce profound quenching in LHCll trimers under hydrostatic pressure ${ }^{56}$. This would mean however, that the re-optimized average structure obtained from the crystals somehow captured the quenched conformation rather than the unquenched population. This explanation therefore seems less likely. 
The quenching mechanism we have captured is rather a general one in which any coupling between the chlorophyll and carotenoids leads to some dissipation. While Lut620 is the strongest quencher it is not the sole one and lut621 and even neoxanthin contribute significantly. This is in contradiction to most proposed quenching mechanisms in which a specific pigment and a specific interaction are cited as the quencher. Indeed, more recently it is apparent that there is neither one quencher nor one quenching conformation. Although we have not considered other mechanism such as charge transfer states (and cannot exclude them) if these specific interactions are solely responsible for quenching then the simple pathways featured in our model must be completely abolished in both the quenching and lightharvesting state.

Models based on static structures have proved highly successful at illustrating the chlorophyll dynamics of this protein and it is likely that these dynamics are relatively stable. However, given the unusual sensitivity of the chlorophyll-carotenoid interactions (arising from the twoelectron character of the latter) to small changes in inter-pigment association, the neglect of dynamical fluctuations is a strong limitation. A natural extension to this work is coupling our model to a MD simulation of LHCII in an explicit membrane environment. Knecht et al have shown that the calculated oscillator strength of the carotenoid $S_{1}$ state is extremely sensitive to small changes in bond length alternation ${ }^{57}$. They conclude that geometric effects are of paramount importance when considering this state. An MD study aimed at studying the $\mathrm{S}_{1}$ state will require an extremely well-parameterized force field description of the carotenoid as cage optimization of even a moderate sample of snapshots would be prohibitively expensive.

\section{Conclusion}

A theoretical understanding of the role of the carotenoid $S_{1}$ state in regulatory/protective quenching in plants, is still in its infancy. This model predicts a quenching pathway that is qualitatively similar to that proposed by Ruban et al. ${ }^{5}$, via the incoherent transfer of energy from the Chl a terminal emitter domain to lut620. However, while lut620 is the predominant quencher in our, lut621 and even neo623 also contribute significantly. By considering more realistic pigment geometries and a more realistic description of the $S_{1}$ 'lineshape' we predict a system significantly less quenched than the model of Chemliov et al. ${ }^{17}$ However, the quenching mechanism itself is qualitatively the same. Our quenching model is consistent with the slow economic quenching by a dark state seen in LHCII aggregates and the PSII antenna. However, our model predicts a much shorter lifetime than the 890 ps average lifetimes of LHCII crystals. Importantly, we have shown that whether a carotenoid can act as a quencher is mostly to do with its close interaction with $\mathrm{Chl}$ a. We saw only a small dependence on $\mathrm{S}_{1}$ site energy and no sharp transition between quenched and unquenched states. Our model of quenching is rather general, in which slow and simple energy transfer to the carotenoids results in profound excitation quenching. Although we do not claim to have identified the quenching mechanism we argue that the light-harvesting state must significantly supress (or even completely abolish) these interactions. Given its qualitative similarity to the incoherent, lutein-mediated pathway identified in LHCII aggregates we argue that the mechanism predicted by this model is a promising candidate for a quenching mechanism.

\section{Acknowledgements}

We would like to acknowledge Dr William Bricker for his advice for the use of his TDC code. K.F, V. B. and C.D are supported by Queen Mary University of London and the Leverhulme 
Trust (RPG-2015-337). J.C and L.V were supported by the Research Council of Lithuania (LMT grant no. MIP-080/2015). A. R. would like to acknowledge the grants from The Leverhulme Trust and UK Biotechnology and Biological Sciences Research Council and The Royal Society for the Wolfson Research Merit Award. 


\section{Figures}

Figure 1: A schematic diagram of the LHCll monomer model structure used in our calculations. We have highlighted the 4 chlorophyll-carotenoid domains which are relevant when discussing potential quenching domains. $\delta_{\mathrm{n}}(\AA)$

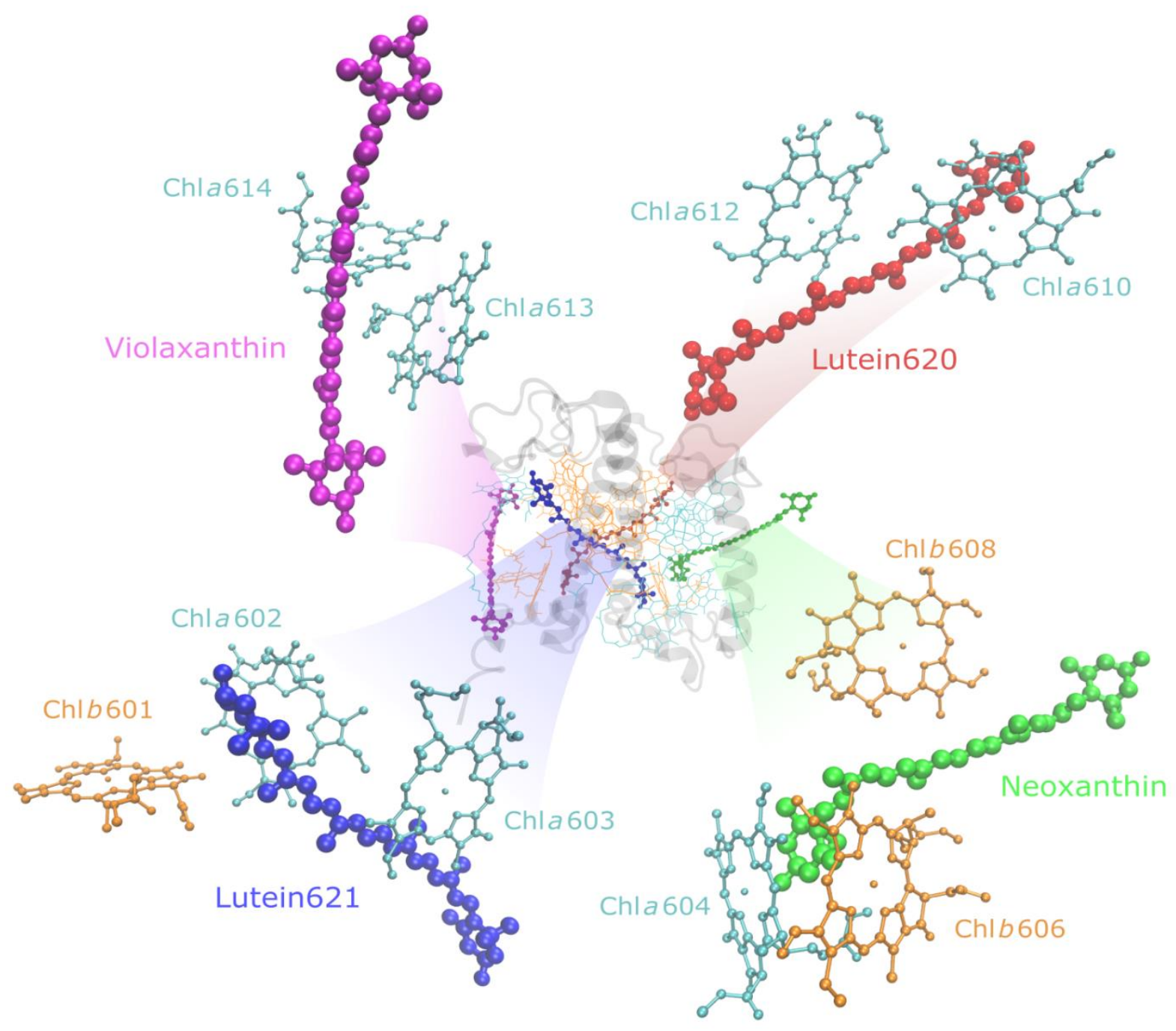


Figure 2 | The fitting process of a spectral density to the two-photon absorption (2PA) of Walla et al. and the $\mathrm{S}_{2}$ absorption (1PA) spectra.

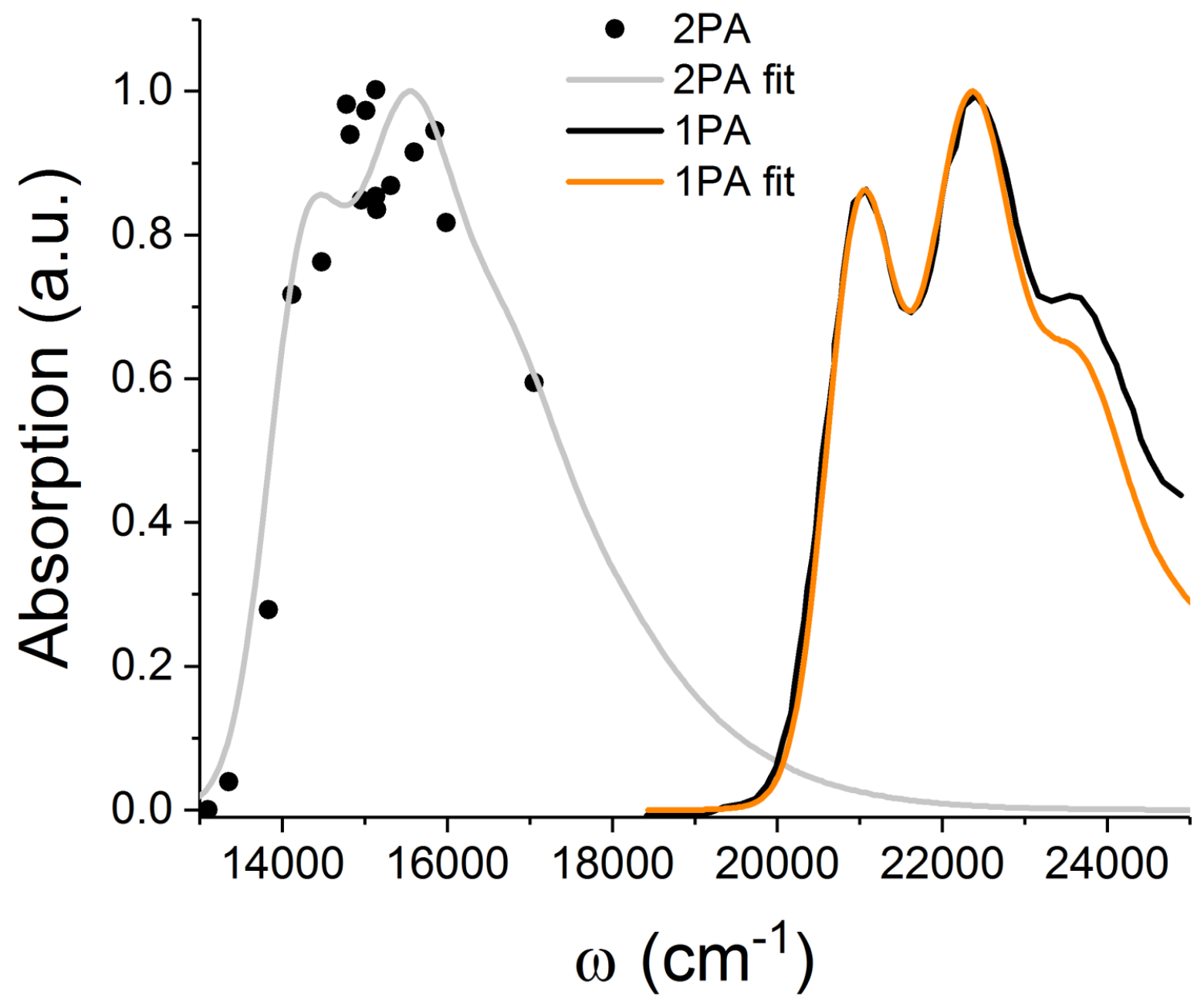


Figure 3 | The hopping times of exciton transfer between pigments. The faded lines represent carotenoid to chlorophyll transfer and the darker lines represent carotenoid to chlorophyll transfer. There is a gap in the $y$-axis as the Neo-Chla604 hopping times are considerably larger than the others presented.

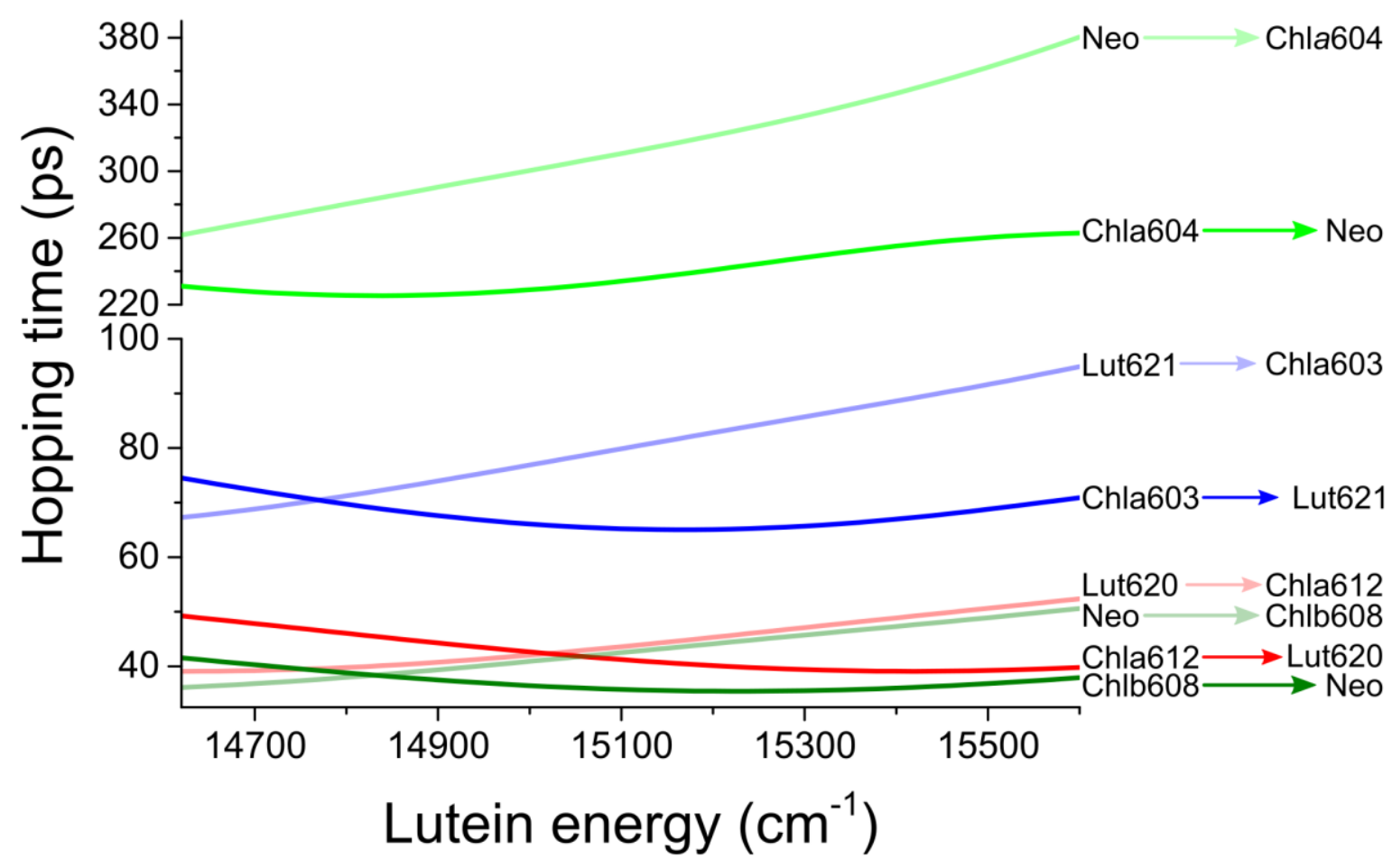


Figure 4 a | The mean excitation lifetime of monomeric LHCII as a function of lut620 site energy. A comparison of the spectral density generated for this paper with the one used by Chmeliov et al. at their reorganisation energy of 3540 and the lowest calculated for a carotenoid at 300 .

$\mathbf{4} \mathbf{b}$ | The relative yields for non-radiative dissipation in monomeric LHCII via different pigment channels as a function of lut620 site energy. The $\mathrm{Chl}$ pool is treated as a whole.
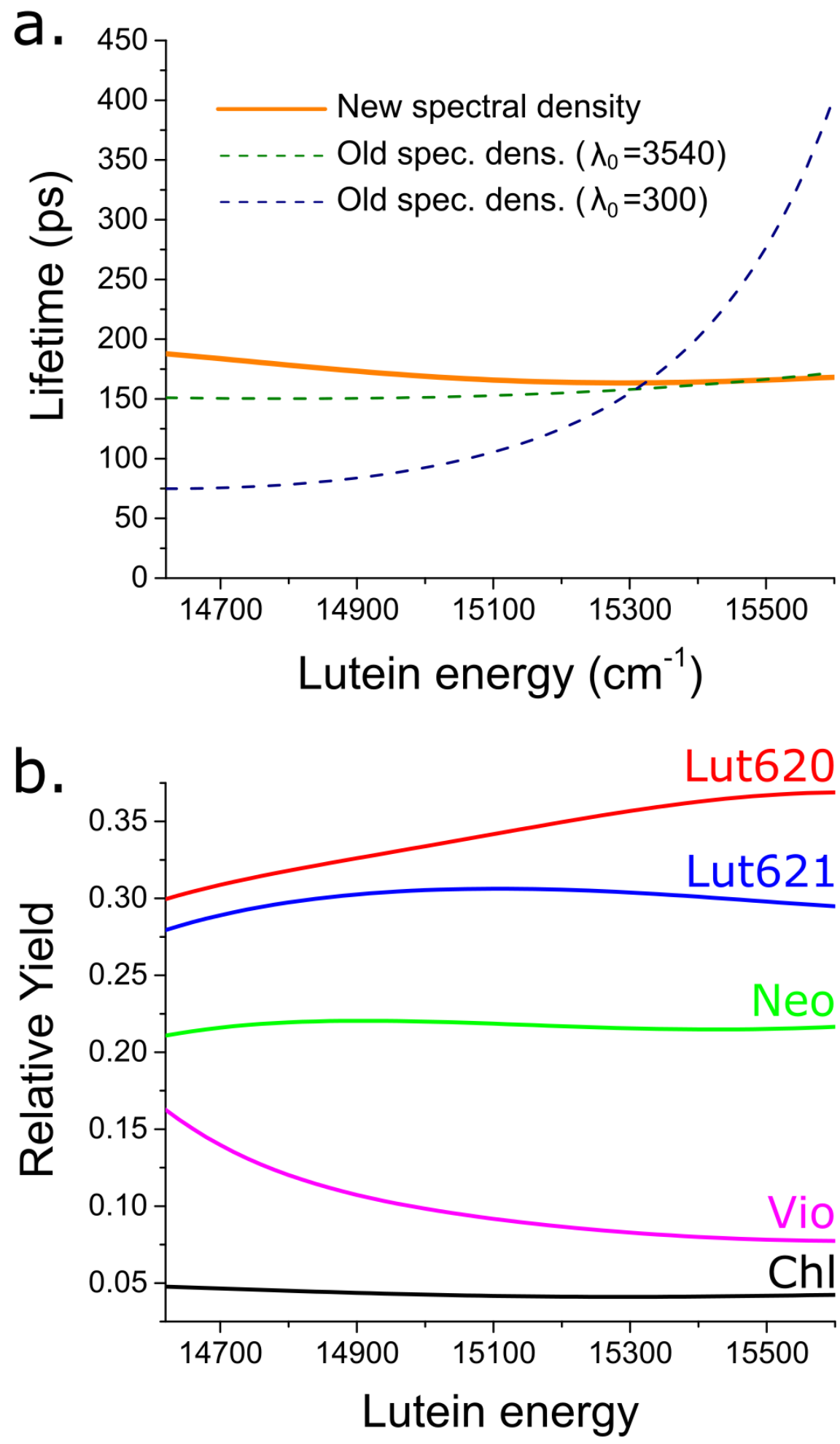
Figure 5 a| The effect of Chl-carotenoid coupling strength on excitation dissipation within LHCII as a function of the carotenoid $S_{1}$ state energy (represented by lut620 site energy) assuming $\lambda_{0}=300 \mathrm{~cm}^{-1}$ : A. Initial couplings. B. All Chl-carotenoid couplings scaled by a factor of 0.2. C. Lut620 uncoupled. D. Lut621 uncoupled. E. Neo623 uncoupled. F. Lut620 and lut621 uncoupled.

5 b| Lifeitme of LHCII as a function of a carotenoid coupling factor. All the carotenoidchlorophyll couplings are treated to a uniform scaling factor, from 0 (disconnecting all carotenoids) to 1 (the calculated couplings).
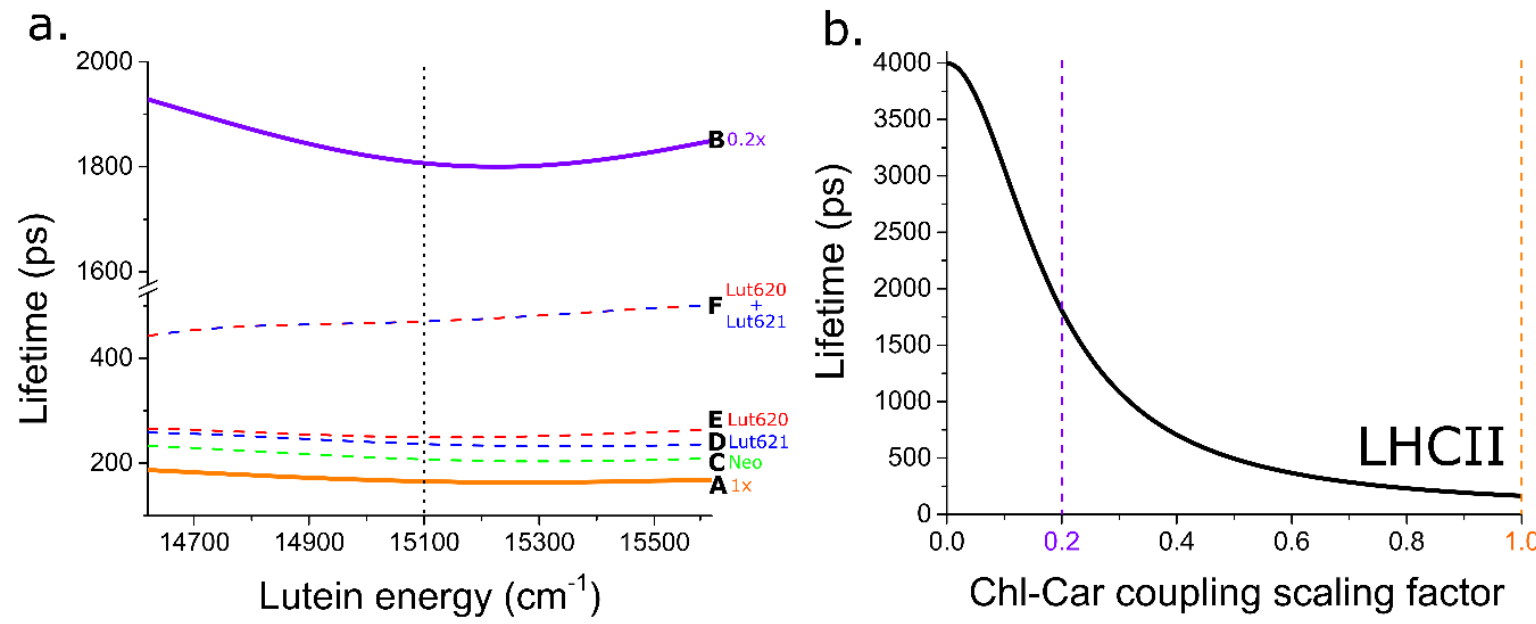


\section{Tables}

Table 1 | A comparison of the calculated and experimental excitation energies and dipole moments. The $S_{1}$ transition of each carotenoid is listed along with average values of the Chl $Q_{y}$ transition, with the numbers in brackets referring to the energies calculated by Andreussi et al. ${ }^{53}$ using DFT/MRCI. The electronic character of each transition is also presented with the carotenoid $S_{1}$ transition being predominantly a double HOMO - LUMO excitation, $\left|\psi_{H O M O_{\uparrow} H O M O_{\downarrow}}^{L U M O_{\downarrow}}\right\rangle$, and the $\mathrm{ChI} \mathrm{Q}_{\mathrm{y}}$ transition being predominantly single HOMO - LUMO, $\left|\psi_{H O M O}^{L U M O}\right\rangle$.

A $S_{1}-S_{2}$ absorption. ${ }^{B} 2$-Photon absorption. ${ }^{C}$ Fluorescence.

\begin{tabular}{|c|c|c|c|c|c|}
\hline & \multicolumn{2}{|l|}{$E_{m}\left(\mathrm{~cm}^{-1}\right)$} & \multicolumn{2}{|l|}{$|\mu|(\mathrm{D})$} & \multirow[t]{2}{*}{ Electronic character } \\
\hline & AM1-CAS-Cl & Exp. & AM1-CAS-Cl & Exp. & \\
\hline Chl a & 16695 & $14884^{9}$ & 7.55 & $4.58^{45}$ & $\left|\psi_{\text {HOMO }}^{L U M O}\right\rangle$ \\
\hline Chl $b$ & 17585 & $15362^{9}$ & 3.63 & $3.83^{45}$ & $\left|\psi_{\text {HOMO }}^{\text {LUMO }}\right\rangle$ \\
\hline Lut620 & $\begin{array}{l}19538 \\
(17180)^{53}\end{array}$ & $14050^{\mathrm{A}}-15300^{\mathrm{B}} 46,55$ & 0.17 & $\sim 0$ & $\left|\psi_{H O M O_{\uparrow} H O M O_{\downarrow}}^{L U M O_{\downarrow} L U M O_{\downarrow}}\right\rangle$ \\
\hline Lut621 & $\begin{array}{l}19748 \\
(17180)^{53}\end{array}$ & $14050-15300$ & 0.30 & $\sim 0$ & $\left|\psi_{H O M O_{\uparrow} H O M O_{\downarrow}}^{L U M O_{\uparrow} L U M O_{\downarrow}}\right|$ \\
\hline Vio622 & $\begin{array}{l}20115 \\
(17583)^{53}\end{array}$ & $13700^{A-15580^{C} 46,57}$ & 0.18 & $\sim 0$ & $\left|\psi_{H O M O_{\uparrow} H O M O_{\downarrow}}^{L U M O_{\downarrow} L U M O_{\downarrow}}\right\rangle$ \\
\hline Neo623 & $\begin{array}{l}20250 \\
(18228)^{53}\end{array}$ & $>15000^{34}$ & 0.15 & $\sim 0$ & $\left|\psi_{H O M O_{\uparrow} H O M O_{\downarrow}}^{L U M O_{\downarrow} L U M O_{\downarrow}}\right\rangle$ \\
\hline
\end{tabular}


Table 2 | The excitonic Hamiltonian (in the site basis). The diagonal elements are the pigment site energies, $E_{m}$. For the Chls, these energies are taken (as in previous work) from the Müh et al. while the carotenoid energies correspond to the calculated AM1-CAS-CI values. The latter are subject to a single variable scaling factor. The off-diagonal elements represent the inter-pigment couplings, $J_{m n}$. The upper right half lists the couplings of Chmeliov et al. ${ }^{17}$ while the lower left half lists the new couplings calculated following cage optimization. For visual clarity, table cells are coloured based on the corresponding coupling strength. The Chl-Chl and Chl-carotenoid couplings are coloured separately since the latter are an order of magnitude smaller than the former.

\begin{tabular}{|c|c|c|c|c|c|c|c|c|c|c|c|c|c|c|c|c|c|c|c|}
\hline Pigments & 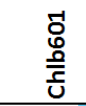 & 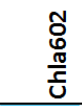 & $\begin{array}{l}n \\
0 \\
\frac{0}{\pi} \\
\frac{\pi}{U}\end{array}$ & 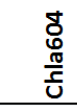 & 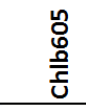 & 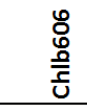 & 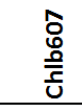 & $\begin{array}{l}\infty \\
0 \\
0 \\
0 \\
\text { 언 }\end{array}$ & 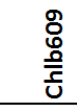 & 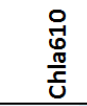 & 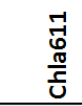 & 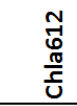 & 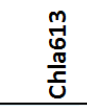 & \begin{tabular}{l}
$\Delta$ \\
\multicolumn{6}{|c}{} \\
$\frac{\pi}{5}$ \\
\end{tabular} & 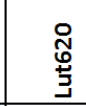 & 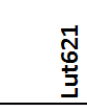 & $\begin{array}{l}\text { స్ } \\
\stackrel{0}{>} \\
\end{array}$ & 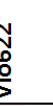 & $\begin{array}{l}m \\
\tilde{N} \\
0 \\
\dot{d} \\
z\end{array}$ \\
\hline Chlb601 & 15405 & -36 & -5 & -2 & 0 & 2 & -2 & -2 & -2 & -4 & -9 & 3 & -7 & -3 & 0 & 0 & 0 & 1 & 0 \\
\hline Chla602 & -20 & 14940 & 18 & 6 & -1 & -6 & 5 & 5 & 18 & -10 & -2 & 11 & -6 & -2 & 0 & -3 & 3 & 0 & 0 \\
\hline Chlab03 & -3 & 26 & 14850 & 5 & -1 & -10 & 1 & 3 & 63 & -9 & -1 & 2 & 1 & -7 & 0 & 11 & 1 & 0 & 0 \\
\hline Chla604 & 2 & -4 & -9 & 14820 & -3 & 78 & -18 & -3 & -7 & 5 & -2 & 0 & 0 & -3 & 1 & -5 & 5 & 0 & 4 \\
\hline Chlb605 & 0 & 0 & 0 & 6 & 15465 & 11 & 1 & -3 & 0 & -2 & 1 & 2 & 1 & 0 & 0 & 0 & 0 & 0 & -1 \\
\hline Chlb606 & 2 & -5 & 2 & 62 & -23 & 15385 & 13 & 3 & 13 & -3 & 2 & 3 & 2 & 2 & 0 & 0 & 2 & 0 & -4 \\
\hline Chlb607 & 1 & -3 & -4 & 7 & -3 & 10 & 15225 & -3 & -6 & -1 & -2 & -2 & -1 & -3 & 0 & -2 & 2 & 0 & 0 \\
\hline Chlb608 & -2 & 4 & -2 & -6 & -1 & -11 & -2 & 15215 & -14 & 40 & -3 & -1 & -1 & -1 & 0 & 0 & 0 & 0 & -3 \\
\hline Chlb609 & 3 & -4 & 88 & -4 & 0 & -16 & 1 & -19 & 15475 & -1 & -3 & -1 & -1 & -2 & 0 & 1 & 1 & 0 & 1 \\
\hline Chla610 & 3 & -9 & -6 & -1 & 1 & -1 & 0 & 30 & 1 & 14790 & -30 & -12 & -7 & -2 & 4 & 0 & 0 & 0 & 0 \\
\hline Chla611 & -14 & 9 & 1 & -2 & 1 & -2 & -1 & 4 & -2 & -17 & 14950 & 131 & -6 & -4 & 1 & 0 & 0 & 0 & 0 \\
\hline Chla612 & 0 & -8 & -1 & 0 & -1 & 3 & 1 & -3 & 0 & 3 & 89 & 14940 & 4 & 1 & 12 & -1 & 1 & 0 & 1 \\
\hline Chla613 & -4 & 10 & 0 & 0 & 1 & -1 & 0 & 1 & -1 & -4 & 1 & -4 & 14840 & -60 & -3 & 0 & 0 & 3 & 0 \\
\hline Chla614 & 2 & -9 & -4 & 2 & 0 & 2 & 1 & -1 & 1 & 1 & -1 & 1 & -18 & 14940 & -1 & 0 & 0 & 2 & 0 \\
\hline Lut620 & 0 & -1 & 0 & 0 & 0 & 0 & 0 & 0 & 0 & 3 & 1 & 6 & -1 & 0 & 19538 & & 0 & 0 & 0 \\
\hline Lut621 & 1 & -2 & 7 & -1 & 0 & 0 & -1 & 0 & 1 & 0 & 0 & -1 & 0 & 0 & & 19748 & & 0 & 0 \\
\hline Vio622 & 1 & -1 & 0 & 0 & 0 & 0 & 0 & 0 & 0 & 0 & 1 & 0 & 3 & 5 & 0 & 0 & \multicolumn{2}{|c|}{020115} & 0 \\
\hline Neo633 & 0 & 0 & 0 & 6 & 0 & -7 & 0 & -9 & 0 & -1 & 0 & 0 & 0 & 0 & 0 & 0 & 0 & & 0250 \\
\hline
\end{tabular}




\section{References}

1. S. B. Powles, Annual Review of Plant Physiology and Plant Molecular Biology, 1984, 35, 15-44.

2. A. V. Ruban, M. P. Johnson and C. D. P. Duffy, Biochimica Et Biophysica Acta-Bioenergetics, 2012, 1817, 167-181.

3. N. E. Holt, D. Zigmantas, L. Valkunas, X. P. Li, K. K. Niyogi and G. R. Fleming, Science, 2005, 307, 433-436.

4. A. A. Pascal, Z. F. Liu, K. Broess, B. van Oort, H. van Amerongen, C. Wang, P. Horton, B. Robert, W. R. Chang and A. Ruban, Nature, 2005, 436, 134-137.

5. A. V. Ruban, R. Berera, C. Ilioaia, I. H. M. van Stokkum, J. T. M. Kennis, A. A. Pascal, H. van Amerongen, B. Robert, P. Horton and R. van Grondelle, Nature, 2007, 450, 575-U522.

6. T. K. Ahn, T. J. Avenson, M. Ballottari, Y. C. Cheng, K. K. Niyogi, R. Bassi and G. R. Fleming, Science, 2008, 320, 794-797.

7. M. G. Muller, P. Lambrev, M. Reus, E. Wientjes, R. Croce and A. R. Holzwarth, Chemphyschem, 2010, 11, 1289-1296.

8. V. Novoderezhkin, A. Marin and R. van Grondelle, Phys. Chem. Chem. Phys., 2011, 13, 1709317103.

9. F. Muh and T. Renger, Biochimica Et Biophysica Acta-Bioenergetics, 2012, 1817, 1446-1460.

10. C. Curutchet and B. Mennucci, Chemical Reviews, 2017, 117, 294-343.

11. Z. F. Liu, H. C. Yan, K. B. Wang, T. Y. Kuang, J. P. Zhang, L. L. Gui, X. M. An and W. R. Chang, Nature, 2004, 428, 287-292.

12. V. I. Novoderezhkin, M. A. Palacios, H. van Amerongen and R. van Grondelle, J. Phys. Chem. B, 2005, 109, 10493-10504.

13. F. Muh, M. E. A. Madjet and T. Renger, J. Phys. Chem. B, 2010, 114, 13517-13535.

14. H. A. Frank, A. Cua, V. Chynwat, A. Young, D. Gosztola and M. R. Wasielewski, Photosynthesis Research, 1994, 41, 389-395.

15. Y. Z. Ma, N. E. Holt, X. P. Li, K. K. Niyogi and G. R. Fleming, Proceedings of the National Academy of Sciences of the United States of America, 2003, 100, 4377-4382.

16. T. Polivka, J. L. Herek, D. Zigmantas, H. E. Akerlund and V. Sundstrom, Proceedings of the National Academy of Sciences of the United States of America, 1999, 96, 4914-4917.

17. J. Chmeliov, W. P. Bricker, C. Lo, E. Jouin, L. Valkunas, A. V. Ruban and C. D. P. Duffy, Physical chemistry chemical physics : PCCP, 2015, 17, 15857-15867.

18. E. Belgio, M. P. Johnson, S. Juric and A. V. Ruban, Biophysical Journal, 2012, 102, 2761-2771.

19. C. C. Gradinaru, I. H. M. van Stokkum, A. A. Pascal, R. van Grondelle and H. van Amerongen, The Journal of Physical Chemistry B, 2000, 104, 9330-9342.

20. V. Barzda, V. Gulbinas, R. Kananavicius, V. Cervinskas, H. van Amerongen, R. van Grondelle and L. Valkunas, Biophysical Journal, 2001, 80, 2409-2421.

21. D. I. G. Bennett, K. Amarnath and G. R. Fleming, Journal of the American Chemical Society, 2013, 135, 9164-9173.

22. J. Chmeliov, G. Trinkunas, H. van Amerongen and L. Valkunas, Journal of the American Chemical Society, 2014, 136, 8963-8972.

23. L. Valkunas, G. Trinkunas, J. Chmeliov and A. V. Ruban, Phys. Chem. Chem. Phys., 2009, 11, 7576-7584.

24. E. Belgio, E. Kapitonova, J. Chmeliov, C. D. P. Duffy, P. Ungerer, L. Valkunas and A. V. Ruban, Nature Communications, 2014, 5, 8.

25. K. F. Fox, W. P. Bricker, C. Lo and C. D. P. Duffy, J. Phys. Chem. B, 2015, 119, 15550-15560.

26. H. Yan, P. Zhang, C. Wang, Z. Liu and W. Chang, Biochem Biophys Res Commun, 2007, 355, 457-463.

27. D. Kroner and J. P. Gotze, Journal of Photochemistry and Photobiology B-Biology, 2012, 109, 12-19.

28. J. P. Götze, D. Kröner, S. Banerjee, B. Karasulu and W. Thiel, ChemPhysChem, 2014, 15, $3392-$ 3401. 
29. S. K. L. Valkunas, G. Juzeliunas, Sov. Phys. Collections, 1985, 26, 54-60.

30. P. J. Walla, P. A. Linden, K. Ohta and G. R. Fleming, Journal of Physical Chemistry A, 2002, 106, 1909-1916.

31. V. Perlik, J. Seibt, L. J. Cranston, R. J. Cogdell, C. N. Lincoln, J. Savolainen, F. Sanda, T. Mancal and J. Hauer, Journal of Chemical Physics, 2015, 142, 15.

32. N. Christensson, F. Milota, A. Nemeth, J. Sperling, H. F. Kauffmann, T. Pullerits and J. Hauer, J. Phys. Chem. B, 2009, 113, 16409-16419.

33. V. Balevicius, A. Gelzinis, D. Abramavicius and L. Valkunas, J. Phys. Chem. B, 2013, 117, $11031-$ 11041.

34. T. Polivka and V. Sundstrom, Chemical Reviews, 2004, 104, 2021-2071.

35. A. D. Becke, Journal of Chemical Physics, 1993, 98, 5648-5652.

36. P. C. Haharan and J. A. Pople, Theor. Chim. Acta, 1973, 28, 213.

37. J. J. P. Stewart, Journal of Molecular Modeling, 2007, 13, 1173-1213.

38. M. J. S. Dewar, E. G. Zoebisch, E. F. Healy and J. J. P. Stewart, Journal of the American Chemical Society, 1985, 107, 3902-3909.

39. J. J. P. Stewart, Collorado Springs, CO, USA, 2012.

40. T. Kusumoto, D. Kosumi, C. Uragami, H. A. Frank, R. R. Birge, R. J. Cogdell and H. Hashimoto, Journal of Physical Chemistry A, 2011, 115, 2110-2119.

41. M. Macernis, J. Sulskus, C. D. P. Duffy, A. V. Ruban and L. Valkunas, Journal of Physical Chemistry A, 2012, 116, 9843-9853.

42. C. D. Duffy, J. Chmeliov, M. Macernis, J. Sulskus, L. Valkunas and A. V. Ruban, J Phys Chem B, 2013, 117, 10974-10986.

43. W. P. Bricker and C. S. Lo, J. Phys. Chem. B, 2015, 119, 5755-5764.

44. B. P. Krueger, G. D. Scholes and G. R. Fleming, J. Phys. Chem. B, 1998, 102, 5378-5386.

45. R. S. Knox and B. Q. Spring, Photochemistry and Photobiology, 2003, 77, 497-501.

46. T. Polivka, D. Zigmantas, V. Sundstrom, E. Formaggio, G. Cinque and R. Bassi, Biochemistry, 2002, 41, 439-450.

47. G. Renger, Current Science, 2010, 98, 1305-1319.

48. T. Renger and R. A. Marcus, Journal of Chemical Physics, 2002, 116, 9997-10019.

49. V. Balevicius, A. G. Pour, J. Savolainen, C. N. Lincoln, V. Lukes, E. Riedle, L. Valkunas, D. Abramavicius and J. Hauer, Phys. Chem. Chem. Phys., 2015, 17, 19491-19499.

50. D. A. L. Valkunas, T Mancal, Molecular Excitation Dynamics and Relaxation, WILEY-VCH Verlag GmbH and Co. KGaA, Weinheim, Germany, 1st edn., 2013.

51. A. V. Ruban, A. A. Pascal, B. Robert and P. Horton, J. Biol. Chem., 2001, 276, 24862-24870.

52. K. Schulten, I. Ohmine and M. Karplus, Journal of Chemical Physics, 1976, 64, 4422-4441.

53. O. Andreussi, S. Knecht, C. M. Marian, J. Kongsted and B. Mennucci, Journal of Chemical Theory and Computation, 2015, 11, 655-666.

54. I. G. Prandi, L. Viani, O. Andreussi and B. Mennucci, Journal of Computational Chemistry, 2016, 37, 981-991.

55. P. J. Walla, J. Yom, B. P. Krueger and G. R. Fleming, J. Phys. Chem. B, 2000, 104, 4799-4806.

56. N. Liguori, X. Periole, S. J. Marrink and R. Croce, Scientific Reports, 2015, 5, 10.

57. J. S. Josue and H. A. Frank, Journal of Physical Chemistry A, 2002, 106, 4815-4824. 